\title{
Dolor pélvico crónico postincisión Pfannenstiel
}

\author{
Pío Iván Gómez Sánchez*
}

\begin{abstract}
RESUMEN: Se presenta un caso de dolor pélvico crónico (DPC) de origen parietal, por atrapamiento de nervio ileohipogástrico, posterior a una incisión de Pfannenstiel. El dolor apareció ocho meses después de la cirugía. Se estudia la paciente en la Clínica de Dolor (CD), del Instituto Materno Infantil (IMI) de Santafé de Bogotá, diagnosticándose el atrapamiento y realizándose un bloqueo nervioso, desapareciendo la sintomatología.
\end{abstract}

Se explica la técnica utilizada y se dan indicaciones para evitar la lesión nerviosa en las incisiones transversas bajas abdominales.

PALABRAS CLAVES: Pfannenstiel, atrapamiento nervioso, bloqueo anestésico.

SUMMARY: A chronic pelvic pain of parietal origin appears due to the entrapment of the ileohypogastric nerve, posterior to the Pfannenstiel's incision.

The pain appeared 8 months after the surgery. The pacient is studied in the Clinic for Pain in the «Instituto Materno Infantil», Bogotá. The entrapment is diagnosed and a nervous blockage is done, disappearing the symptomatology. The technique applied is explained and indications are given to avoid nervous lesions in the lower abdominal transverse incisions.

KEY WORDS: Pfannenstiel incision, nerve entrapment, anestesic blockage.

\section{Introducción}

Una de las causas de DPC de origen parietal, es el atrapamiento, en su trayecto de nervios de la pared abdominal, ya sea espontáneamente, lo que es raro, o luego de incisiones transversas en la pared abdominal, como las utilizadas en herniorrafias, apendicectomías, y la tan frecuentemente utilizada en Ginecobstetricia, incisión de Pfannenstiel (1). Este tipo de atrapamiento, rara vez diagnosticado, por desconocimiento, tanto de su etiopatogenia, como de su tratamiento, tiende a ser subvalorado en las pacientes que lo padecen.

\section{Reporte de un caso}

Paciente de 35 años, quien consulta a la Clínica de Dolor (CD) del Instituto Materno Infantil (IMI), por dolor en el hipogastrio de ocho meses de evolución, tipo peso y quemante, que se iniciaba en la cicatriz quirúrgica de una cesárea realizada un año atrás, mediante incisión de Pfannenstiel. El dolor se irradiaba a la región inguinal y labio mayor derechos.

Según la escala numérica subjetiva verbal, la paciente refería el dolor con una intensidad de 7/10.

Valorada en la CD, se encuentra una paciente con un examen físico general normal, encontrando como hallazgo positivo, dolor a la palpación profunda, sobre el tercio externo derecho de la cicatriz quirúrgica, que no dismi-

* Profesor asistente. Universidad Nacional. Coordinador Clínica de Dolor. Instituto Materno Infantil. nuía al hacer tensionar los músculos de la pared abdominal.

La anamnesis no mostró alteraciones de la esfera sexual; los paraclínicos (bioquímica sanguínea, uroanálisis y ultrasonido pélvico), estaban dentro de la normalidad.

La evaluación por Psicología, no encontró alteraciones mayores y sólo anotó insomnio desde la aparición del dolor.

Se hace impresión diagnóstica de atrapamiento de nervio ileohipogástrico y se decide practicar bloqueo anestésico del mismo, de acuerdo con el esquema de la figura 1, con introducción de una aguja raquídea No. 22 , en el punto marcado con una «X», localizado $2.5 \mathrm{cms}$ por dentro y 2.5 cms por debajo de la espina iliaca anterosuperior, haciendo tres infiltraciones en la aponeurosis; la primera, perpendicular al borde de los músculos rectos abdominales, la segunda, dirigida al tubérculo púbico y la tercera equidistante a las dos anteriores (ver Figura 1).

Teniendo en cuenta que, la infiltración podía aliviar el dolor por efecto placebo, se decidió practicar la infiltración con agua destilada estéril; la infiltración reprodujo inmediatamente el dolor, siendo referido por la paciente como intenso y al no haber mejoría del mismo en la semana siguiente, se decidió realizar una segunda infiltración, con igual técnica, pero esta vez con Bupivacaína al $0.5 \%, 10 \mathrm{cc}$, aplicando $1 \mathrm{cc}$ en el sitio de la punción inicial, y luego $3 \mathrm{cc}$ en cada una de las tres infiltraciones descritas (igual cantidad e igual proporción de agua destilada, se utilizó en la primera infiltración).

Inicialmente, la paciente refirió incremento del dolor, pero a los 10 minutos hubo mejoría, desapareciendo por completo el dolor en los 30 minutos siguientes. 
Se controló la paciente, semanalmente, por dos meses y luego trimestralmente, llevando hasta el momento once meses de seguimiento con mejoría completa.

\section{Figura 1}

LOCALIZACION NERVIOS ILEOHIPOGASTRICO E ILEOINGUINAL. PUNTO DE BLOQUEO (X).

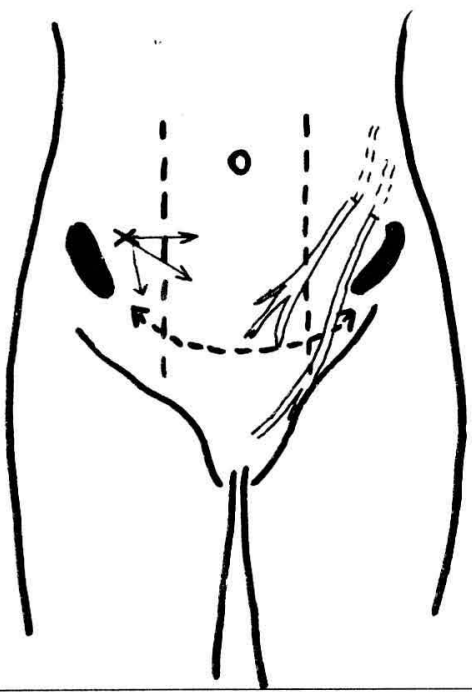

$\mathrm{X}$ : Punto de bloqueo.

$--->$ : Direcciones de la aguja.

-.--: Incisión de Pfannenstiel.

: Bordes de músculos rectos abdominales.

$\mathrm{O}$ : Ombligo.

\section{Discusión}

El atrapamiento nervioso postincisión de Pfannenstiel, puede ser causado por: incluir el nervio al saturar la aponeurosis, por tracción del mismo al terminar el proceso de cicatrización que puede producir retracción de los tejidos y en última instancia por lesión directa nerviosa y formación de un neuroma (2).

La sintomatología se caracteriza por dolor que puede aparecer inmediatamente después de la cirugía e inclusive meses o años más tarde.

El dolor puede ser descrito como vago, localizado en la zona baja abdominal, como una sensación quemante en la cicatriz quirúrgica o dolor tipo peso, que se puede irradiar a la cara anterior del muslo y/o región vulvar.

El diagnóstico se basa en una historia clínica detallada y en un examen físico completo, tendiente a descartar otras etiologías y enfatizando en la utilidad semiológica de no disminuirse el dolor a la palpación, cuando se tensionan los músculos abdominales, lo que si sucede cuando la causa del dolor es intrabdominal.

El bloqueo mediante la técnica descrita puede liberar a la paciente de los síntomas en forma permanente o transitoria, con la posibilidad de repetir el bloqueo varias veces. Cuando se requieren muchos bloqueos y el dolor reaparece, se debe proceder a seccionar quirúrgicamente el nervio (3).

La lesión del nervio ileohipogástrico se puede evitar si en lo posible, la incisión aponeurótica del oblicuo externo, no se extiende más allá del borde externo de los rectos abdominales y no tomando mucho tejido en los ángulos de la aponeurosis. En conclusión el bloqueo nervioso descrito, puede ser útil en pacientes que tengan un cuadro similar al descrito y si hay mejoría, pueden evitarse procedimientos diagnósticos o terapéuticos invasivos y costosos.

\title{
BIBLIOGRAFIA
}

1. Gómez PI. Dolor pélvico crónico en Ginecología. Boletín Clínica de Dolor Pélvico Crónico. Instituto Materno Infantil de Bogotá. 1990; vol. 1 y 2.

2. Grosz CR. Iliohypogastric Nerve Injury. Am. J. Surg. 1981; vol. 628.
3. Sippo WC., Burghardt A., Gomez A. Nerve Entrapment after Pfannenstiel Incision. Am. J. Obstet. Gynecol. 1987; 157: 420-421.

\section{LA REVISTA COLOMBIANA DE OBSTETRICIA Y GINECOLOGIA}

\author{
A N U N C I A
}

Premio anual al mejor trabajo científico publicado en la revista durante 1994

Nota: Las bases de concurso se publicarán en la revista No. 1 Vol. 45 de 1994 